\title{
Searches for Exotic Higgs-like boson decays at $\mathrm{LHCb}$
}

\author{
L. Sestini, on behalf of the LHCb collaboration* \\ INFN Padova \\ E-mail: lorenzo.sestini@cern.ch
}

\begin{abstract}
$\mathrm{LHCb}$ has made a series of searches sensitive to Higgs-like bosons and their decays. These analyses include searches for long-lived particles produced in the decays of such resonances, with these particles decaying to various final states probed in different analyses, and a search for Higgs-like bosons that produce lepton-flavour-violating decays, producing a tau mu final state. The unique design of the $\mathrm{LHCb}$ detector, with a flexible trigger and a precise vertex detector, enables competitive and world-best limits, particularly for low mass states, and for states that have a low lifetime. These results, along with future prospects, are presented.
\end{abstract}

European Physical Society Conference on High Energy Physics - EPS-HEP2019 -

10-17 July, 2019

Ghent, Belgium

${ }^{*}$ Speaker. 


\section{Introduction}

In the search for New Physics (NP) two different approaches are followed by the LHC experiments. The first one is the indirect search, where deviations from the Standard Model (SM) are sought in the properties of known processes, e.g. in rare $B$ mesons decays. The second approach is the direct search for NP particles by reconstructing their decays to SM particles. The LHCb experiment [1] has the capability to pursue both strategies. In this contribution the latest LHCb results on the direct search for exotic Higgs-like boson decays are presented. In particular two different decay topologies are discussed: the decay of long-lived particles, with decay vertices significantly displaced from the $p p$ collision primary vertex, and the lepton flavour violating decays, where leptons of different flavours (e.g. muon and tau) are produced.

LHCb [1] is a forward spectrometer, initially designed to study $b$ and $c$ quarks. Among the LHC experiments, LHCb alone provides precision coverage in the forward region of $p p$ collisions corresponding to the $2 \leq \eta \leq 5$ pseudo-rapidity range. LHCb has an excellent tracking system composed of a Vertex Locator (VELO) and tracking stations: thanks to an excellent impact parameter (IP) and momentum resolution it can measure a $100 \mathrm{ps}$ lifetime with a precision of $0.2 \mathrm{ps}$. LHCb has also a particle identification system, formed by two RICHes, two calorimeters (ECAL and HCAL) and a muon system: the muon identification efficiency is $97 \%$ with a pion misidentification of $1-3 \%$.

\section{Long-lived particles decaying semileptonically}

LHCb searched for long-lived particles decaying semileptonically in data collected during the LHC Run 1, in collisions with a center of mass energy of $\sqrt{s}=7,8 \mathrm{TeV}$, corresponding to an integrated luminosity of $3 \mathrm{fb}^{-1}$ [2]. This search is motivated by several NP models, as example the $R$-parity violating mSugra neutralino model, where the Higgs boson decays to long-lived neutralinos that can decay semileptonically. The signature of this search is a Secondary Vertex (SV) with at least one high transverse momentum $\left(p_{\mathrm{T}}\right)$ muon track significantly displaced from the Primary Vertex (PV), with $p_{\mathrm{T}}>12 \mathrm{GeV}$ and $I P>0.25$. Collisions that have a SV with a distance from the beam axis greater than $0.55 \mathrm{~mm}$ are selected. The signal yield has been extracted with a fit to the SV invariant mass, testing several signal masses and lifetimes. In the fit the models for signal and background (mainly $b \bar{b}$ ) are obtained with simulation. An example of fit result with a particular signal hypothesis is shown in figure 1 . No signal is observed and upper limits on the cross sections are established with the CLs method. The expected and observed limits at 95\% Confidence Level (CL) as a function of the neutralino mass and for two different lifetime hypotheses are shown in figure 2 .

\section{Long-lived particles decaying to jet pairs}

LHCb searched for long-lived particles decaying to jet pairs in data collected during the LHC Run 1 , in collisions with $\sqrt{s}=7,8 \mathrm{TeV}$, corresponding to an integrated luminosity of $3 \mathrm{fb}^{-1}$ [3]. This search is motivated by Hidden Valley models, where the Higgs boson can decay in longlived Hidden Valley pions that subsequently decay in $b \bar{b}$ pairs. The signature of this search is two 


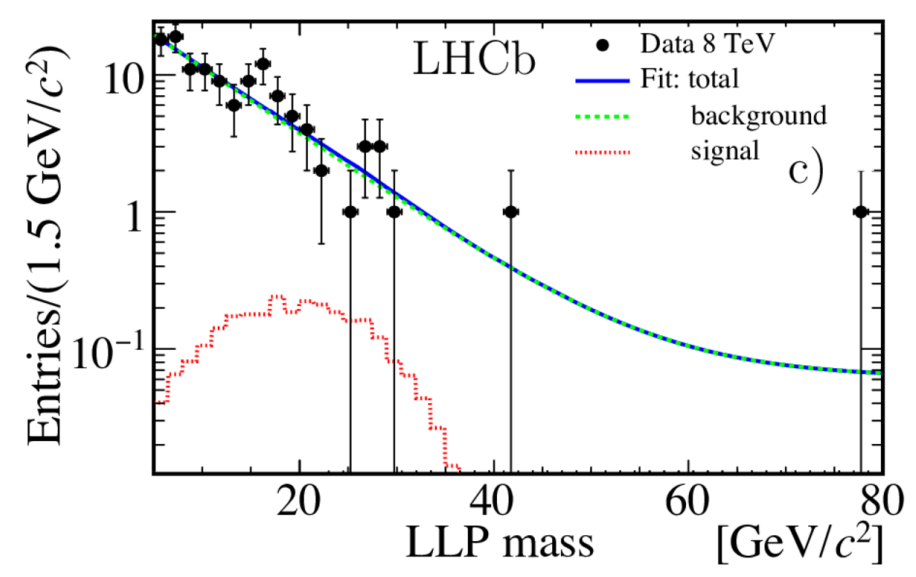

Figure 1: Fit to the invariant mass distribution of the SV, used to extract the signal yield of long-lived neutralinos decaying semileptonically. A particular signal hypothesis is shown.
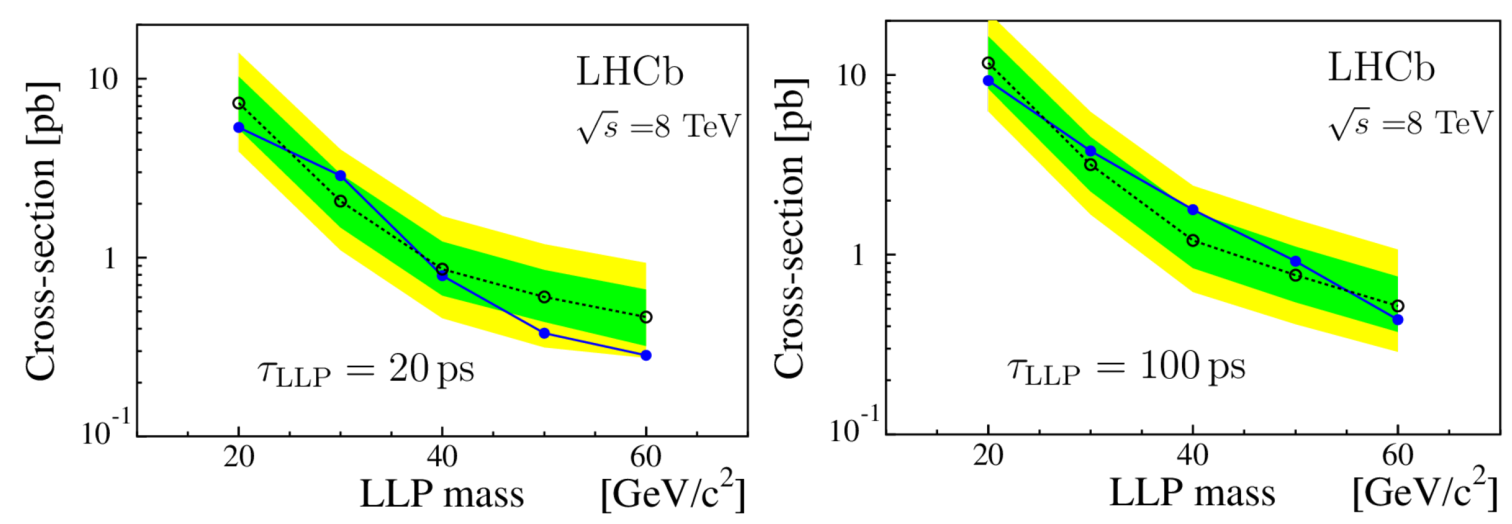

Figure 2: Expected and observed upper limits at $95 \% \mathrm{CL}$ on the production cross section of a long-lived neutralino decaying semileptonically, as a function of the neutralino mass and for two different lifetime hypotheses.

reconstructed jets associated to a displaced SV. Jets have been reconstructed with a particle flow algorithm with anti-kt clustering and a cone radius of $R=0.7$. Collisions with two jets associated to a SV with a distance from the beam axis greater than $0.4 \mathrm{~mm}$ are selected. The signal yield has been extracted with a fit to the dijet invariant invariant mass distribution, testing several signal masses and lifetimes. Several fits have been perfomed in different intervals of SV displacement from the beam axis, to improve the sensitivity. In the fits models for both signal and background (mainly $b \bar{b}$ ) have been obtained with simulation. An example of fit result with particular signal hypothesis and SV displacement interval is shown in figure 3. No signal is observed and upper limits on the production cross sections are established with the CLs method. The expected and observed limits at $95 \% \mathrm{CL}$ as a function of the pion mass and lifetime are shown in figure 4. The LHCb exclusion region is compared with that of ATLAS and CMS in figure 5, showing the complementarity of $\mathrm{LHCb}$ with respect to the other LHC experiments. The limits are competitive despite a factor 10 lower integrated luminosity. 


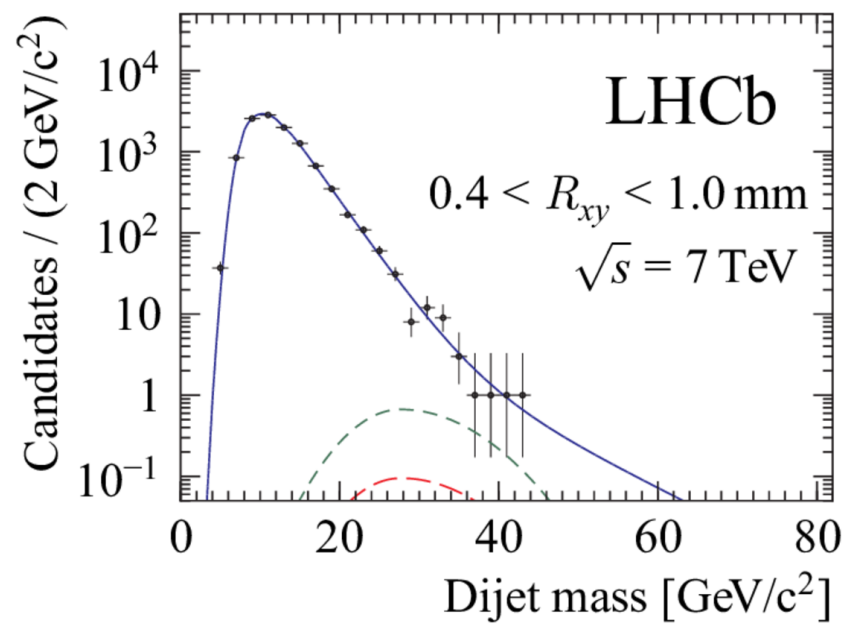

Figure 3: Fit to the dijet invariant mass distribution, used to extract the signal yield of long-lived pion decaying in two jets. A particular signal hypothesis and SV displacement $R_{x y}$ interval are shown.

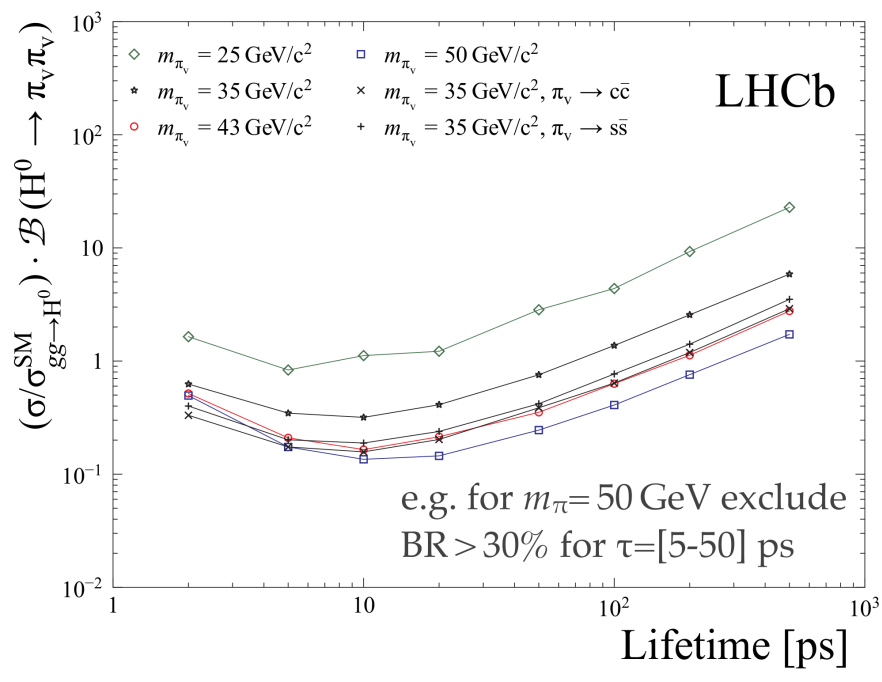

Figure 4: Expected and observed upper limits at $95 \% \mathrm{CL}$ on the production cross section of a Hidden Valley pion decaying to jets, as a function of the pion mass and lifetime.

\section{Higgs lepton flavour violating decays}

LHCb set upper limits on the decay of the Higgs into a muon and a tau lepton using data collected during the LHC Run 1 at $\sqrt{s}=8 \mathrm{TeV}$, corresponding to an integrated luminosity of 2 $\mathrm{fb}^{-1}$ [4]. The signature of this search is a prompt muon track and a tau candidate. Tau leptons are reconstructed in four different channels, corresponding to their different decay modes: a muon displaced with respect to the PV, a displaced hadron, a displaced electron or a SV formed from 3 displaced hadrons. The background yield is estimated from predicted cross sections and simulation, 


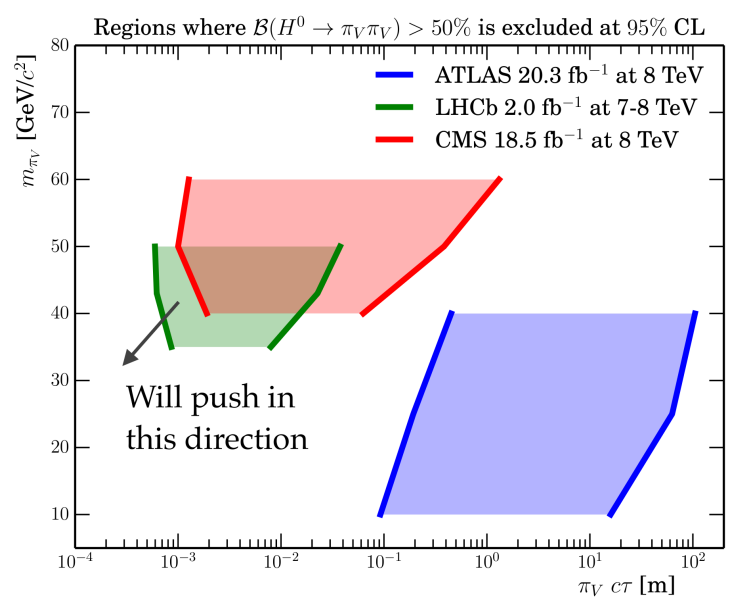

Figure 5: Comparison of the LHCb exclusion region for the Branching Ratio of $H \rightarrow \pi_{v} \pi_{v}$, where $\pi_{v}$ is a long-lived particle decaying to jets, with respect to ATLAS and CMS.

the main contribution is given by the $Z \rightarrow \tau \tau$ process. The number of observed signal events is obtained by subtracting the number of expected background events from the number of selected events. An example of an invariant mass distribution comparison between data and expectation is presented in figure 6. No signal is observed and upper limits on the cross sections are enstablished with the CLs method. The expected and observed limits at $95 \% \mathrm{CL}$ as a function of the Higgs mass and lifetime are shown in figure 7. The results are also shown separately for the different decay channels. For the SM Higgs the upper limit on the $H \rightarrow \mu \tau$ Branching Ratio is $26 \%$.

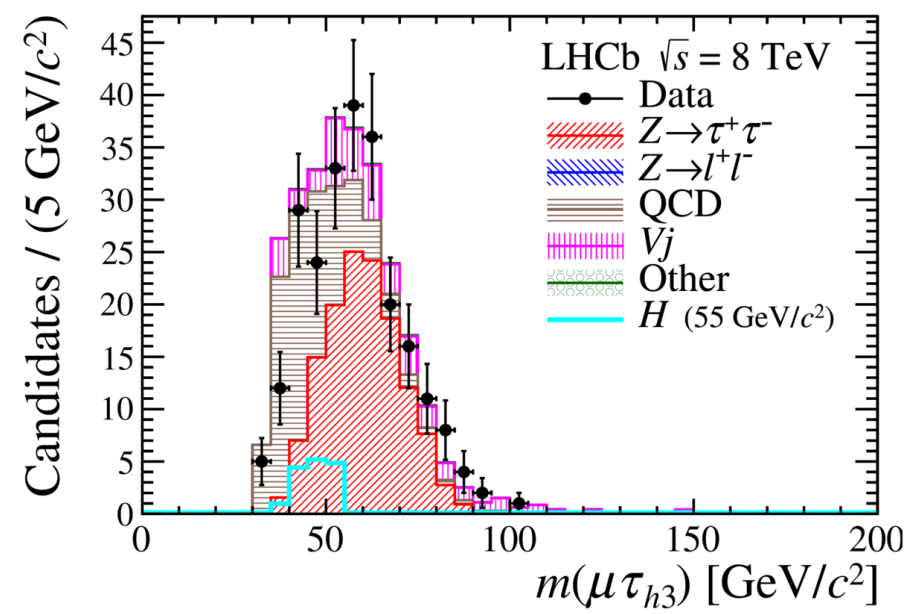

Figure 6: Invariant mass distribution of $H \rightarrow \mu \tau$ candidates, where the tau lepton is reconstructed in the three-hadron final state. Data distribution is compared with the background expectation.

\section{Prospects for long-lived particles searches at LHCb upgrades}

Extrapolated sensitivities of future LHCb searches for long-lived particles have been made in 


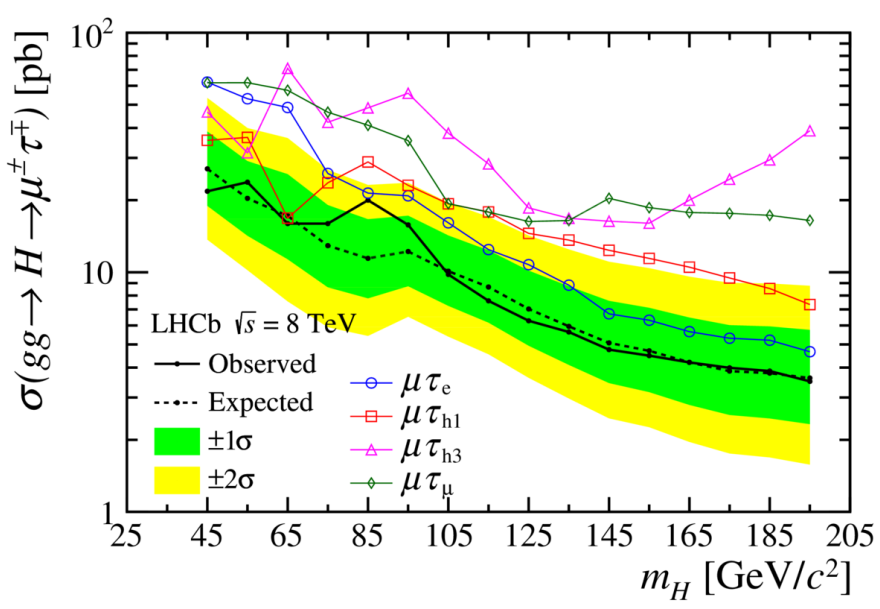

Figure 7: Expected and observed upper limits at 95\% CL on the production cross section of $H \rightarrow \mu \tau$, as a function of the Higgs mass.

[5]. Several realistic assumptions on the upgraded detector and data-taking system have been made to evaluate the improvement on the selection efficiencies. An upgraded LHCb detector for the HLLHC phase is expected to record an integrated luminosity of $300 \mathrm{fb}^{-1}$ by the end of Run 5 . In this scenario, the expected limit on the branching ratio of the Higgs decaying to a pair of neutralinos, excluded at $95 \% \mathrm{CL}$, as a function of the neutralino lifetime and mass is shown in figure 8 . This has been obtained starting from the results reported in Section 2. Similarly, the results reported in Section 3 have been used to determine the minimum Branching Ratio for the Higgs decaying to a pair of hidden valley pions, excluded at $95 \% \mathrm{CL}$, as a function of the hidden vallet pion lifetime and mass. The extrapolation is shown in figure 9.
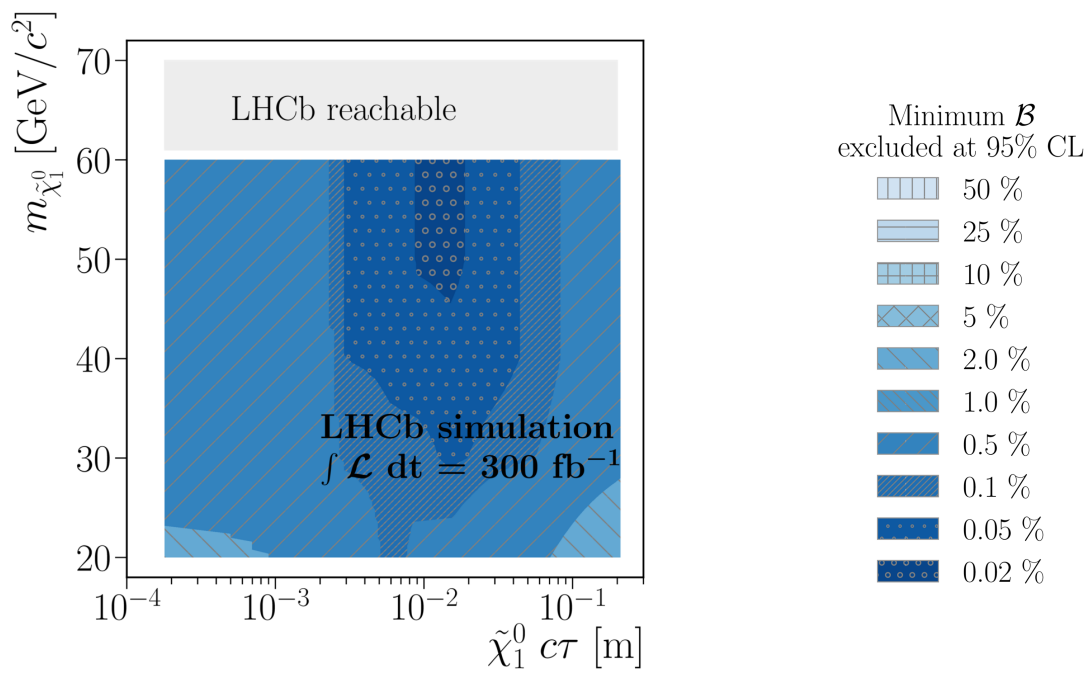

Figure 8: Extrapolated exclusion regions for the branching ratio of the Higgs decaying to a pair of neutralinos at $300 \mathrm{fb}^{-1}$. 

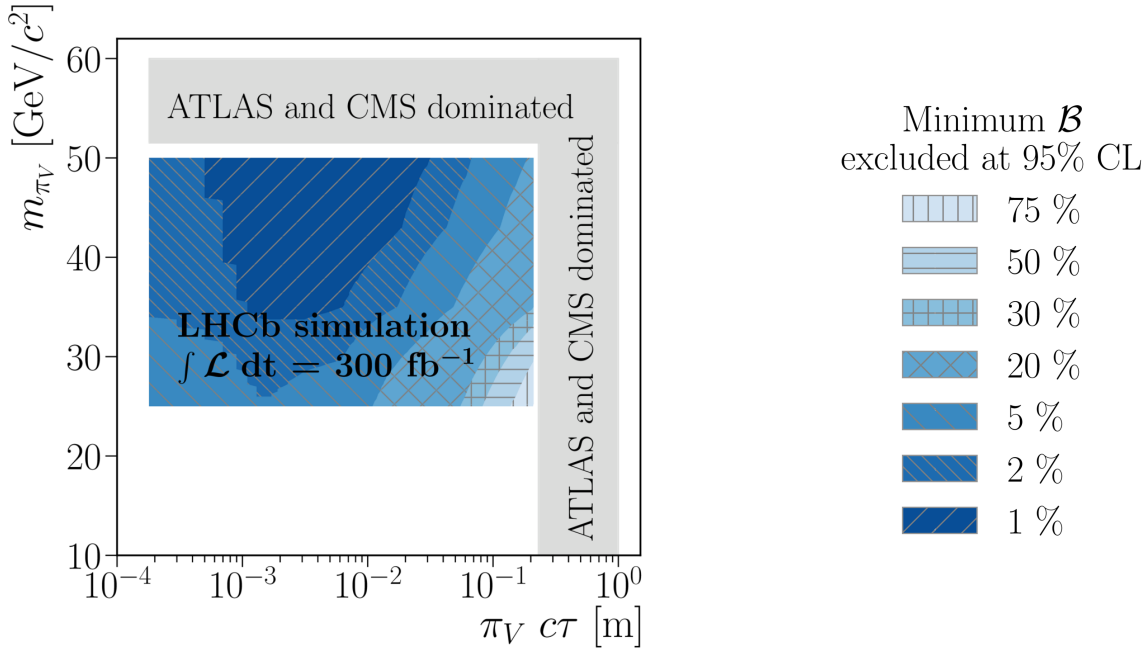

Figure 9: Extrapolated exclusion regions for the branching ratio of the Higgs decaying to a pair of hidden valley pions at $300 \mathrm{fb}^{-1}$.

\section{Conclusions}

In this contribution the latest $\mathrm{LHCb}$ results on the direct search for exotic Higgs-like boson decays have been reported. These searches are complementary to ATLAS and CMS, thanks to the phase space region covered by LHCb. Expected sensitivities of future long-lived particle searches in the HL-LHC era have been reported, showing that LHCb promises interesting sensitivity in direct searches for NP.

\section{References}

[1] A. A. Alves Jr. et al., The LHCb detector at LHC, JINST 3 (2008) S08005.

[2] LHCb collaboration, Search for massive long-lived particles decaying semileptonically in the LHCb detector, Eur. Phys. J. C77 (2017) 224.

[3] LHCb collaboration, Updated search for long-lived particles decaying to jet pairs, Eur. Phys. J. C77 (2017) 812.

[4] LHCb collaboration, Search for lepton-flavour-violating decays of Higgs-like bosons, Eur. Phys. J. C78 (2018) 1008.

[5] LHCb collaboration, Prospects for searches for long-lived particles after the LHCb detector upgrades, LHCb-CONF-2018-006. 